\title{
Determinants of Disability in Rheumatoid Arthritis: A Community-Based Cohort Study
}

\author{
Megan L. Krause ${ }^{1}$, Cynthia S. Crowson ${ }^{2}$, Tim Bongartz ${ }^{4}$, Eric L. Matteson ${ }^{1,3}$, Clement J. Michet ${ }^{1}$, \\ Thomas G. Mason ${ }^{1}$, Scott T. Persellin ${ }^{1}$, Sherine E. Gabriel ${ }^{1,3}$ and John M. Davis III ${ }^{*}, 1$
}

${ }^{I}$ Division of Rheumatology, Department of Medicine; ${ }^{2}$ Division of Biomedical Statistics and Informatics, Department of Health Sciences Research; ${ }^{3}$ Division of Epidemiology, Department of Health Sciences Research, College of Medicine, Mayo Clinic, Rochester, Minnesota, USA; ${ }^{4}$ Department of Emergency Medicine, Vanderbilt University Medical Center, Nashville, TN, USA

\begin{abstract}
Longitudinal care of a community-based cohort of patients with rheumatoid arthritis (RA) was evaluated retrospectively. Candidate determinants of disability included visual analog scales (VAS) for patient global assessment and pain, comorbidities, and medications. The outcome was the 'patient-acceptable symptom state' for disability as defined by the Health Assessment Questionnaire (HAQ) disability index, using a cutoff of $<1.04$. Two-sample $t$ tests and multivariable logistic regression were used to determine odds ratios (OR) for associations between predictor variables and disability. Out of a total of 99 patients, $28(28 \%)$ patients had HAQ $\geq 1.04$ at their last visit. The greatest odds of not attaining the patient-acceptable symptom state in a multivariable model was associated with corticosteroids (OR: 5.1; $\mathrm{p}=0.02$ ), antidepressants (OR: $5.3 ; \mathrm{p}=0.02$ ), and female sex (OR: 6.5; $\mathrm{p}=0.05$ ). In the era of biologic therapy, female sex, corticosteroids, and antidepressants remain profound determinants of disability highlighting the need to understand the underlying mechanisms.
\end{abstract}

Keywords: Depression, disability, rheumatoid arthritis.

\section{INTRODUCTION}

In the era of increasing use of biologic therapy and data regarding comparative effectiveness of treatment regimens, patients continue to experience significant disability secondary to rheumatoid arthritis (RA). While there is evidence of declining overall disability in large cohorts, disability continues to be prominent and serves as a frequent cause of permanent loss of employment [1-3]. Even in the ideal setting of clinical trials with aggressive therapy regimens, the impact of RA on disability is not eliminated [4-6].

Cohort studies of patients with RA demonstrate the importance of baseline health assessment questionnaire (HAQ), erosions, autoantibody status, swollen joint counts, and age as predictors of future disability [7-9]. Further, the importance of comorbidity on disability progression has been emphasized in a large RA cohort, particularly cardiovascular disease and higher overall burden of comorbidities [10]. In these cohort studies, data were obtained at baseline and then at different subsequent time points; however it is often unclear if data such as HAQ or patient assessments of disease were available in real time to clinicians to allow for adjustment in management. Further, these cohorts are variable in terms of the utilization of biologic therapies due in part to the time of publication before high rates of biologic use. In addition to the medical features, socioeconomic status

*Address correspondence to this author at the Division of Rheumatology, Mayo Clinic, 200 First St. SW, Rochester, MN 55905, USA;

Fax: 507-284-0564; E-mail: davis.john4@mayo.edu and psychosocial factors such as learned helplessness serve as potential mediators of future disability; however it is unclear how these factors acted upon by clinicians if at all [11-13].

The goal of this study was to evaluate a communitybased cohort of persons with RA with ready access to biologic therapy for patient-centered determinants of disability. This study utilized the Rochester Epidemiology Project (REP), a unique cohort that allows for retrospective evaluation of clinical data that was obtained during routine clinical care [14]. As this data was generated as part of routine clinical care, it was available to clinicians in real time to make adjustments to the management strategy for individual patients. Understanding the factors that drive disability is relevant not only in contemplating intensification of therapy, but also in considering nonimmune approaches of improving function. Even if inflammation is controlled, other disease-associated factors may need to be modified to optimize physical function. This comprehensive approach will allow for a high quality, patient-centered management plan.

\section{MATERIALS AND METHODS}

\section{Patient Cohort}

A retrospective chart review of a random sample of 100 patients with RA as part of a population-based cohort, utilizing the REP RA cohort including a total of 501 patients, was performed [14]. The information obtained from the chart review was used in the care of patients and was available to 
clinicians at the point-of-care. This study included patients fulfilling the 1987 American College of Rheumatology (ACR) classification criteria for RA [15]. In order to evaluate for change in disability and disease activity, eligible patients must have been evaluated by a rheumatologist on 3 occasions such that the index visit occurred after $1 / 1 / 2009$ with requirement for a second visit within 12 months followed by a third visit within six to eighteen months of the previous visit. Patients were followed until 3/1/2012.

Baseline characteristics, including age, sex, race, ethnicity, education level, alcoholism, smoking status, current employment status, and current relationship status, were identified based on patient provided information. Other information regarding comorbidities was obtained, including clinical diagnoses of anxiety, depression, fibromyalgia, or obstructive sleep apnea by chart review including clinical notes and diagnosis lists. Diabetes mellitus was defined by physician diagnosis and/or documented use of insulin and/or oral hypoglycemic agents [16].

Information regarding the date of RA diagnosis, extraarticular manifestations of RA, radiographic evidence of erosions, and history of joint surgery were identified [17]. Serologic status including rheumatoid factor (RF) and anticitrullinated protein antibodies (ACPA) were identified including any history of positive results and the most recent value to index date.

As part of all visits to the Inflammatory Arthritis Clinic, patients complete a global assessment of disease activity on a visual analog scale (VAS; 0-100 mm), patient pain assessment VAS $(0-100 \mathrm{~mm})$, and HAQ (0-3) prior to the appointment, hence this data was available to their rheumatologists in real time to assist in management decision-making [18]. Providers' assessments, including swollen and tender joint counts (0-28 joints), global VAS (0$100 \mathrm{~mm})$, and qualitative assessment of disease activity (active versus inactive), were abstracted if the rheumatologist had documented these during their visit. Inflammatory markers (ESR, CRP) were abstracted at each visit when available.

Baseline medications specific to RA were determined at the index appointment. Both previous and current use of methotrexate, other disease modifying antirheumatic drugs (DMARDs) (including hydroxychloroquine, sulfasalazine, and leflunomide), tumor necrosis factor ( $\mathrm{TNF} \alpha$ ) inhibitors, and other biologics (including abatacept, rituximab, and tocilizumab) were identified. Corticosteroid use was recorded as current use ( $\leq 4$ weeks), recent use ( $>4$ to $\leq 12$ weeks), or no recent use ( $>12$ weeks). At each appointment, changes to medications were recorded in the form of start, increase, discontinue, decrease, or no change for the categories methotrexate, other DMARDs, TNF $\alpha$ inhibitors, other biologics, or corticosteroids. Identified side effects related to medications were also recorded. Information regarding the timing and number of joint injections was also abstracted. Use of adjuvant medications, including opioids, tramadol, anxiolytics, antidepressants, muscle relaxants, pregabalin/gabapentin, and sleep aids, were evaluated at each appointment.

\section{Data Analysis}

Study data were recorded and managed using the Research Electronic Data Capture system [19]. Research Electronic Data Capture is a secure, web-based application designed to support data capture for research studies.

For the descriptive statistics, continuous variables were described using mean \pm standard deviation (SD), and categorical variables were described with percentages. The "patient-acceptable symptom state" for physical disability was defined by the HAQ disability index using a cutoff of $<1.04$ [20]. The range of HAQ is $0-3$ with $<1$ felt to represent mild disability and $<2$ moderate disability [21]. This patient-acceptable symptom state was identified in a cohort of RA patients using a question regarding satisfactory condition both with $75^{\text {th }}$ percentile estimation as well as receiver operating characteristic analysis. Two-sample t tests and multivariable logistic regression were used to determine odds ratios (OR) for associations between predictor variables and disability.

\section{RESULTS}

\section{Patient Characteristics}

One hundred patients of the population cohort were evaluated, and HAQ data were available for 99 . The mean age at first visit was $59.9 \pm 14.0$, with $76.8 \%$ females. The mean duration of RA at the first index visit was $9.5 \pm 6.4$ years. RF status was available in 95 of 99 patients, with $82.1 \%$ positive for RF. The ACPA status was available in $74 / 99$ and positive in $54.1 \%$. Joint erosions visualized on $\mathrm{x}-$ ray and/or MRI were present in $63.6 \%$, and $34.3 \%$ had undergone at least one joint surgery. The patients were followed for a median of 2.5 years with range of 0.6-3.2 years. The mean value of the patient global VAS at the index visit was $28 \pm 24$. At the final visit, the patient VAS global was $31 \pm 26$.

\section{Factors Predictive of Patient-Acceptable State at Final Visit}

In this cohort, 28 of 99 patients $(28 \%)$ did not attain the patient-acceptable symptom state for physical disability as defined by HAQ $<1.04$ at their last visit. In terms of baseline characteristics, older patients, women, and patients who were not actively employed were more likely to have elevated final HAQ values. Higher initial HAQ and patient global VAS both at the index and final visit were associated with an elevated HAQ at the concluding visit. Joint surgery was associated with higher disability at patients' last visit. Increased number of tender joints, but not of swollen joints, was significantly associated with not reaching the patientacceptable symptom state. Previous use of methotrexate and recent or increasing corticosteroid use were associated with elevated HAQ values. No prior use of other DMARDs was also associated with failing to attain the patient-acceptable state. Use of antidepressants, anxiolytics, or tramadol/opioids was associated with an elevated HAQ value (Table 1). 
Table 1. Characteristics associated with final patient-acceptable symptom state.

\begin{tabular}{|c|c|c|c|}
\hline Variable & $\begin{array}{c}\text { HAQ }<1.04 \\
(N=71)\end{array}$ & $\begin{array}{c}\text { HAQ } \geq 1.04 \\
(\mathrm{~N}=28)\end{array}$ & p-Value \\
\hline Age at first visit, years & $57 \pm 13.1$ & $67.5 \pm 13.6$ & 0.003 \\
\hline Duration of RA, years & $9.2(6.3)$ & $10.2(6.6)$ & 0.372 \\
\hline RF positive status & $57(83.8 \%)$ & $21(77.8 \%)$ & 0.488 \\
\hline ESR & $13.8(11.0)$ & $17.0(13.9)$ & 0.313 \\
\hline CRP & $12.3(26.8)$ & $15.8(23.1)$ & 0.152 \\
\hline Patient global VAS (mm), first visit & $22.4 \pm 21.9$ & $41.7 \pm 21.9$ & $<0.001$ \\
\hline Pain VAS (mm), first visit & $29.8 \pm 24.6$ & $44.4 \pm 22.6$ & 0.008 \\
\hline Other DMARD use, ever & $63(88.7 \%)$ & $20(71.4 \%)$ & 0.035 \\
\hline Biologic use & $25(35.2 \%)$ & $15(53.6 \%)$ & 0.094 \\
\hline Corticosteroid use & $20(28.2 \%)$ & $17(60.7 \%)$ & 0.003 \\
\hline Any medication start/increase & $52(73.2 \%)$ & $23(82.1 \%)$ & 0.352 \\
\hline Tramadol/opioid use & $25(35.2 \%)$ & $17(60.7 \%)$ & 0.021 \\
\hline Antidepressant use & $14(19.7 \%)$ & $13(46.4 \%)$ & 0.007 \\
\hline Anxiolytic use & $3(4.2 \%)$ & $8(28.6 \%)$ & 0.001 \\
\hline Depression & $21(29.6 \%)$ & $12(42.9 \%)$ & 0.207 \\
\hline
\end{tabular}

Pertinent factors not predictive of disability included autoantibody status, inflammatory markers at index visit, duration of RA, and smoking status. Comorbidities including diabetes mellitus, fibromyalgia, and obstructive sleep apnea were also not associated with disability. Current use or initiation of TNF inhibitors or other biologic therapies were not associated with the final patient-acceptable symptom state. Further, combining all medication initiations and dosage increases as a surrogate of escalation of therapy was also not associated with the final patient-acceptable symptom state.

Multivariable logistic regression was performed utilizing a model both with and without baseline HAQ (Table 2). When baseline HAQ was in the model, age and the absence of other DMARD use were significantly associated with decreased likelihood that the patient reached an acceptable symptom state, HAQ $\geq 1.04$. If baseline HAQ was removed from the model, corticosteroid use (OR 5.1), antidepressant use (OR 5.3), and female sex (OR 6.5) were the most strongly associated with failure to reach the patient-acceptable symptom state.

\section{Factors Associated with Antidepressant Use}

Twenty-seven patients were utilizing antidepressants during this study. Patients who used antidepressants were less likely to be ACPA positive. These patients were more likely to have a higher tender joint count. They were more likely to have undergone joint surgery. Further, patients who used antidepressants were more likely to use an anxiolytic or sleep aid. There was no difference in terms of duration of RA, employment status, joint erosions, or use of RA-specific medications (Table $\mathbf{3}$ ).

\section{Physician Assessment of Disease Activity}

At the first visit providers felt that disease was active in $30.3 \%$, which decreased slightly to $27.3 \%$ at the last visit. Of the 30 who were considered to have active disease at the first visit, 11 were considered to have persistently active disease at the last visit. In contrast, among 69 considered initially to have inactive disease, 16 patients were considered to have active disease at the final visit. Among individuals who had a $\mathrm{HAQ} \geq 1.04$ at the final visit, only 3 of $28(10.7 \%)$ were considered to have active disease at both the first and last visit. These 3 patients were all women with nonerosive seronegative arthritis. One of the three was currently smoking. Further 2 had a diagnosis of depression and one of these also had a diagnosis of fibromyalgia.

\section{DISCUSSION}

By evaluating a modern community cohort outside of clinical trials in the setting of increasingly available biologic therapy, this study sought to update our understanding of 
Table 2. Determinants of the final patient-acceptable symptom state.

\begin{tabular}{|c|c|c|c|c|}
\hline Effect With Baseline HAQ & Odds Ratio & \multicolumn{2}{|c|}{$95 \%$ CI } & p-Value \\
\hline Age & 1.073 & 1.015 & 1.133 & 0.0130 \\
\hline Female & 2.591 & 0.350 & 19.231 & 0.3517 \\
\hline Corticosteroid use & 3.094 & 0.677 & 14.144 & 0.1452 \\
\hline Other DMARD use & 0.124 & 0.020 & 0.776 & 0.0258 \\
\hline Tramadol/Opioid use & 3.997 & 0.916 & 17.437 & 0.0652 \\
\hline Antidepressant use & 3.446 & 0.779 & 15.250 & 0.1031 \\
\hline Patient Global VAS at first visit & 1.024 & 0.993 & 1.057 & 0.1321 \\
\hline HAQ at first visit & 5.645 & 1.534 & 20.775 & 0.0092 \\
\hline Effect Without Baseline HAQ & Odds Ratio & \multicolumn{2}{|c|}{$95 \%$ CI } & p-Value \\
\hline Age & 1.075 & 1.023 & 1.130 & 0.0042 \\
\hline Female & 6.494 & 1.04 & 40.0 & 0.0456 \\
\hline Corticosteroid use & 5.099 & 1.290 & 20.159 & 0.0202 \\
\hline Other DMARD use & 0.137 & 0.028 & 0.674 & 0.0145 \\
\hline Tramadol/Opioid use & 3.272 & 0.901 & 11.888 & 0.0717 \\
\hline Antidepressant use & 5.255 & 1.324 & 20.852 & 0.0183 \\
\hline Patient Global VAS at first visit & 1.041 & 1.012 & 1.071 & 0.0055 \\
\hline
\end{tabular}

The results of multivariable logistic regression are shown. The dependent variable was patient-unacceptable disability defined by the final HAQ value $\geq 1.04$.

Table 3. Characteristics associated with antidepressant use.

\begin{tabular}{|c|c|c|c|}
\hline Variable & No Antidepressant (N=73) & Antidepressant (N=27) & p-Value \\
\hline \hline Age at first visit, years & $59.8 \pm 14.6$ & $60.0 \pm 12.4$ & 0.966 \\
\hline Sex (female) & $53(72.6 \%)$ & $24(88.9 \%)$ & 0.086 \\
\hline ACPA positive status & $33(62.3 \%)$ & $8(36.4 \%)$ & 0.040 \\
\hline Joint erosions & $49(67.1 \%)$ & $14(51.9 \%)$ & 0.160 \\
\hline Joint surgery & $19(26 \%)$ & $15(55.6 \%)$ & 0.006 \\
\hline Tender joint count, max & $3.7 \pm 5.9$ & $7.6 \pm 7.1$ & $<002$ \\
\hline Anxiolytic use & $3(4.1 \%)$ & $8(29.6 \%)$ & $<0.001$ \\
\hline Sleep aid use & $6(8.2 \%)$ & $12(44.4 \%)$ & $<0.001$ \\
\hline
\end{tabular}

Values are reported as mean $\pm \mathrm{SD}$ or $\mathrm{N}$ (percentage value).

what factors are associated with disability. Ultimately, preventing disability is a shared goal of patients and providers. Fortunately, disability rates are declining in the biologic era and goals of tight control of inflammation but continue to have significant impact on patients [1-3]. Multiple clinical factors are associated with disability based on previous cohort data, including age, RF positivity, tender joint count, inflammatory markers including ESR and CRP, and joint damage [7-9, 22]. Further, psychosocial factors further modify these clinical factors and contribute to the ultimate experience of disability by the patient $[11,13]$. In our study, age and tender joint count were associated in the univariate analysis with disability while RF status, inflammatory markers, and joint erosions were not.

Our study demonstrated that the use of other DMARDs such as hydroxychloroquine, leflunomide, and sulfasalazine was associated with a lower disability, likely representing patients with low disease severity whose disease did not require further escalation. All patients with an end HAQ $\geq$ 1.04 and a vast majority of those with a lower rating had previously tried methotrexate. The minority with low disability who had not taken methotrexate likely represent the same group of other DMARD users with mild disease severity. Despite the era of biologic therapy, with 39 patients having exposure to TNF $\alpha$ inhibitors and 12 patients having exposure to other biologics, corticosteroid use remained one of the strongest predictors of future disability. Biologic therapy was not associated with the final symptom state which is likely a reflection that this study is longitudinal and not a clinical trial and thus confounding variables may interfere with association. 
Depression has a major impact on patients with RA and is associated with higher patient-reported global disease activity and pain as well as disability and mortality [23-25]. In this study, antidepressant use was associated with final disability status. Interestingly, patients treated with antidepressants were less likely to be ACPA-positive, which has been variably associated with disability in other studies $[26,27]$. While antidepressant use was significantly associated with ultimate HAQ status, the clinical diagnosis of depression was not. This finding could potentially be explained if medication lists are more routinely updated at each follow-up visit than the clinical problem list or past medical history. Alternatively, antidepressant use could be reflective of more severe depression.

Interestingly, a clinical diagnosis of fibromyalgia was not associated with disability as previously demonstrated [28]. However, this is likely due to the relatively low number with a clinical diagnosis of fibromyalgia, 8 patients in the cohort. This number may under-represent the patients with components of peripheral or central pain sensitization because the abstraction required a formal diagnosis of fibromyalgia to be listed in the clinical notes.

Pain sensitization impacts the experience of patients with RA [29]. It may in part explain why escalation of therapy, as represented by initiation or increase in dose, was not associated with disability. In some clinical scenarios, symptoms were not felt to be driven by acute inflammation. This discordance between patients and clinicians has been demonstrated in previous RA cohorts and raises the possibility of pain sensitization as an underlying mechanism $[30,31]$. Further, pain thresholds in patients with RA are known to be associated with depression, which corroborates the finding of an association between antidepressant use and disability [32]. The use of antidepressants in this cohort could speculatively reflect an attempt by clinicians to target patients' pain experience and may explain why antidepressant use and not depression was associated with higher disability rates. Relatively low use of pain modulators such as duloxetine, pregabalin or gabapentin was observed in this study, suggesting that these agents represent an underutilized therapeutic option to manage pain in community dwellers with RA.

This study was limited by its retrospective nature, and there were missing data including joint counts and provider global VAS. Further, the qualitative assessment was dependent on the interpretation of the clinician's documentation, so there was potential for misclassification. The requirement for 3 visits over the course of 3 years potentially may have excluded patients with more severe disability or fewer resources by their inability to follow-up with appointments, which could have reduced the power to identify factors associated with disability.

The strengths of this study included evaluation of a community cohort in the era of biologic therapy. We were able to evaluate diverse factors that could impact disability including demographic data, laboratory data, radiographic data, medication use, patient assessments, and provider assessments. Further, it was a longitudinal study allowing for evaluation of future outcomes with baseline characteristics. This study utilizes a HAQ defined patient acceptable state which has been used by other studies to assess outcomes with importance identified by patients with RA [33]. Further, this technique to generate the patient acceptable state has been expanded upon in other disease such as systemic lupus erythematosus and osteoarthritis $[34,35]$.

Even in the era of biologic therapies, patients' experiences of pain and disease activity, treatment for mood and anxiety disorders, and RA treatment characteristics are still important determinants of disability. The findings underscore the utmost importance of incorporating patient perspectives and disease assessments in discussing options for treatment/prevention of disability. Future research should investigate the underpinnings of persistent corticosteroid use and antidepressant use, which could enlighten new interventions for improvement of outcomes.

\section{CONFLICT OF INTEREST}

Its contents are solely the responsibility of the authors and do not necessarily represent the official views of the NIH.

\section{ACKNOWLEDGEMENTS}

This work was supported by a grant from the National Institutes of Health, NIAMS (R01 AR46849), the Rochester Epidemiology Project (R01 AG034676 from the National Institute on Aging), and grant number UL1 TR000135 from the National Center for Advancing Translational Sciences (NCATS).

\section{REFERENCES}

[1] Krishnan E, Lingala B, Bruce B, Fries JF. Disability in rheumatoid arthritis in the era of biological treatments. Ann Rheum Dis 2012; 71(2): 213-8.

[2] Hallert E, Husberg M, Bernfort L. The incidence of permanent work disability in patients with rheumatoid arthritis in Sweden 1990-2010: before and after introduction of biologic agents. Rheumatology 2012; 51(2): 338-46.

[3] Rantalaiho VM, Kautiainen H, Jarvenpaa S, et al. Decline in work disability caused by early rheumatoid arthritis: results from a nationwide Finnish register, 2000-8. Ann Rheum Dis 2013; 72(5): 672-7.

[4] Moreland LW, O'Dell JR, Paulus HE, et al. A randomized comparative effectiveness study of oral triple therapy versus etanercept plus methotrexate in early aggressive rheumatoid arthritis: the treatment of early aggressive rheumatoid arthritis trial. Arthritis Rheum 2012; 64(9): 2824-35.

[5] Goekoop-Ruiterman YP, de Vries-Bouwstra JK, Allaart CF, et al. Clinical and radiographic outcomes of four different treatment strategies in patients with early rheumatoid arthritis (the BeSt study): a randomized, controlled trial. Arthritis Rheum 2005; 52(11): 3381-90.

[6] Breedveld FC, Weisman $\mathrm{MH}$, Kavanaugh AF, et al. The PREMIER study: A multicenter, randomized, double-blind clinical trial of combination therapy with adalimumab plus methotrexate versus methotrexate alone or adalimumab alone in patients with early, aggressive rheumatoid arthritis who had not had previous methotrexate treatment. Arthritis Rheum 2006; 54(1): 26-37.

[7] Combe B, Cantagrel A, Goupille P, et al. Predictive factors of 5year health assessment questionnaire disability in early rheumatoid arthritis. J Rheumatol 2003; 30(11): 2344-9.

[8] Drossaers-Bakker KW, Zwinderman AH, Vliet Vlieland TP, et al. Long-term outcome in rheumatoid arthritis: a simple algorithm of baseline parameters can predict radiographic damage, disability, and disease course at 12-year followup. Arthritis Rheum 2002; 47(4): 383-90.

[9] Graell E, Vazquez I, Larrosa M, et al. Disability measured by the modified health assessment questionnaire in early rheumatoid 
arthritis: prognostic factors after two years of follow-up. Clin Exp Rheumatol 2009; 27(2): 284-91.

[10] Michaud K, Wallenstein G, Wolfe F. Treatment and nontreatment predictors of health assessment questionnaire disability progression in rheumatoid arthritis: a longitudinal study of 18,485 patients. Arthritis Care Res (Hoboken) 2011; 63(3): 366-72.

[11] Jacobi CE, Mol GD, Boshuizen HC, Rupp I, Dinant HJ, Van den Bos GAM. Impact of socioeconomic status on the course of rheumatoid arthritis and on related use of health care services. Arthrit Rheum-Arthr 2003; 49(4): 567-73.

[12] Marra CA, Lynd LD, Esdaile JM, Kopec J, Anis AH. The impact of low family income on self-reported health outcomes in patients with rheumatoid arthritis within a publicly funded health-care environment. Rheumatology (Oxford) 2004; 43(11): 1390-7.

[13] Camacho EM, Verstappen SM, Symmons DP. Association between socioeconomic status, learned helplessness, and disease outcome in patients with inflammatory polyarthritis. Arthritis Care Res (Hoboken) 2012; 64(8): 1225-32.

[14] Kremers MH, Crowson CS, Gabriel SE. Rochester Epidemiology Project: a unique resource for research in the rheumatic diseases. Rheum Dis Clin North Am 2004; 30(4): 819-34.

[15] Arnett FC, Edworthy SM, Bloch DA, et al. The American Rheumatism Association 1987 revised criteria for the classification of rheumatoid arthritis. Arthritis Rheum 1988; 31(3): 315-24.

[16] Liang KP, Myasoedova E, Crowson CS, et al. Increased prevalence of diastolic dysfunction in rheumatoid arthritis. Ann Rheum Dis 2010; 69(9): 1665-70.

[17] Turesson C, O'Fallon WM, Crowson CS, Gabriel SE, Matteson EL. Occurrence of extraarticular disease manifestations is associated with excess mortality in a community based cohort of patients with rheumatoid arthritis. J Rheumatol 2002; 29(1): 62-7.

[18] Bruce B, Fries JF. The Stanford Health Assessment Questionnaire: a review of its history, issues, progress, and documentation. J Rheumatol 2003; 30(1): 167-78.

[19] Harris PA, Taylor R, Thielke R, Payne J, Gonzalez N, Conde JG. Research electronic data capture (REDCap)--a metadata-driven methodology and workflow process for providing translational research informatics support. J Biomed Inform 2009; 42(2): 37781.

[20] Heiberg T, Kvien TK, Mowinckel P, Aletaha D, Smolen JS, Hagen KB. Identification of disease activity and health status cut-off points for the symptom state acceptable to patients with rheumatoid arthritis. Ann Rheum Dis 2008; 67(7): 967-71.

[21] Fries JF, Spitz P, Kraines RG, Holman HR. Measurement of patient outcome in arthritis. Arthritis Rheum 1980; 23(2): 137-45.

[22] Bombardier C, Barbieri M, Parthan A, et al. The relationship between joint damage and functional disability in rheumatoid arthritis: a systematic review. Ann Rheum Dis 2012; 71(6): 836-44.
[23] Ward MM. Are patient self-report measures of arthritis activity confounded by mood? A longitudinal study of patients with rheumatoid arthritis. J Rheumatol 1994; 21(6): 1046-50.

[24] Morris A, Yelin EH, Panopalis P, Julian L, Katz PP. Long-term patterns of depression and associations with health and function in a panel study of rheumatoid arthritis. J Health Psychol 2011; 16(4): 667-77.

[25] Ang DC, Choi H, Kroenke K, Wolfe F. Comorbid depression is an independent risk factor for mortality in patients with rheumatoid arthritis. J Rheumatol 2005; 32(6): 1013-9.

[26] Shidara K, Inoue E, Hoshi D, et al. Anti-cyclic citrullinated peptide antibody predicts functional disability in patients with rheumatoid arthritis in a large prospective observational cohort in Japan. Rheumatol Int 2012; 32(2): 361-6.

[27] del Val del Amo N, Bosch IR, Manteca FC, Gutierrez Polo R, Cortina LE. Anti-cyclic citrullinated peptide antibody in rheumatoid arthritis: relation with disease aggressiveness. Clin Exp Rheumatol 2006; 24(3): 281-6.

[28] Wolfe F, Michaud K. Severe rheumatoid arthritis (RA), worse outcomes, comorbid illness, and sociodemographic disadvantage characterize RA patients with fibromyalgia. J Rheumatol 2004; 31(4): 695-700.

[29] Meeus M, Vervisch S, De Clerck LS, Moorkens G, Hans G, Nijs J. Central sensitization in patients with rheumatoid arthritis: a systematic literature review. Semin Arthritis Rheum 2012; 41(4): 556-67.

[30] Barton JL, Imboden J, Graf J, Glidden D, Yelin EH, Schillinger D. Patient-physician discordance in assessments of global disease severity in rheumatoid arthritis. Arthritis Care Res (Hoboken) 2010; 62(6): 857-64.

[31] Nicolau G, Yogui MM, Vallochi TL, Gianini RJ, Laurindo IM, Novaes GS. Sources of discrepancy in patient and physician global assessments of rheumatoid arthritis disease activity. J Rheumatol 2004; 31(7): 1293-6.

[32] Pollard LC, Ibrahim F, Choy EH, Scott DL. Pain thresholds in rheumatoid arthritis: the effect of tender point counts and disease duration. J Rheumatol 2012; 39(1): 28-31.

[33] Strand V, Smolen JS, van Vollenhoven RF, et al. Certolizumab pegol plus methotrexate provides broad relief from the burden of rheumatoid arthritis: analysis of patient-reported outcomes from the RAPID 2 trial. Ann Rheum Dis 2011; 70(6): 996-1002.

[34] Conti F, Ceccarelli F, Massaro L, et al. Evaluation of the patient acceptable symptom state (PASS) in Italian patients affected by systemic lupus erythematosus: association with disease activity indices. PLoS One 2013; 8(9): e73517.

[35] Maxwell JL, Felson DT, Niu J, et al. Does clinically important change in function after knee replacement guarantee good absolute function? The multicenter osteoarthritis study. J Rheumatol 2014; 41(1): 60-4.

(C) Krause et al.; Licensee Bentham Open.

This is an open access article licensed under the terms of the Creative Commons Attribution Non-Commercial License (http://creativecommons.org/licenses/by-nc/ 4.0/) which permits unrestricted, non-commercial use, distribution and reproduction in any medium, provided the work is properly cited. 